\title{
Visual Servoing Path Tracking for Safe Human-Robot Interaction
}

\author{
G. J. Garcia, J. A. Corrales, J. Pomares, F. A. Candelas and F. Torres \\ Physics, Systems Engineering and Signal Theory Department \\ University of Alicante \\ PO Box 99, 03080, Alicante, Spain \\ \{gjgg, jcorrales, jpomares, francisco.candelas,fernando.torres\}@ua.es
}

\begin{abstract}
When a human is introduced into a robotic cell, the robot controller must be aware of the human location in order to assure her/his physical integrity. This paper presents a pre-collision strategy which maintains a safety distance between a robot and a human who wears a tracking system composed of a motion capture suit and a UWB localization system. The system proposed is able to guide the robot using visual servoing through a previously defined path. The time-independent behaviour of this system enables the robot to completely track the desired trajectory, even in those cases when the robot goes away from the human during the tracking to guarantee her/his safety.
\end{abstract}

Keywords: visual servoing; human tracking; image path tracking; motion capture; human-robot interaction

\section{INTRODUCTION}

Human safety is the first requirement to be fulfilled when human operators and robotic manipulators collaborate in industrial environments. Some previous research about this topic has only tried to reduce the effect of collisions by mechanical protection devices [1][2] or by motion controllers that reduce the impact force [3][4]. However, these post-collision strategies are not appropriate for industrial workplaces because they permit collisions to occur and thus unwanted accidents may happen to humans. Pre-collision strategies avoid possible collisions by changing the path of the robot when a person is nearby [5][6][7]. Nevertheless, this path re-planning process does not maintain the original path and a new trajectory is established each time a person approaches the robot.

The main aim of the pre-collision strategy presented in this paper is to make the robot complete the original planned path after the danger of collision has disappeared. Initially, the robot tracks a previously defined path in the image space using visual servoing. During the tracking of this path, if the human approaches too close to the robot, the robot will move away from her/him to maintain a safety distance. However, after the danger of collision has disappeared, most image trajectory tracking methods based on visual servoing fail [8][9][10][11] to return to the initial path because they are time-dependent. During the presence of the human, the controller of time-dependent tracking methods continues sending time variable references. Once the human detection ends, the robot does not track correctly the original trajectory because it tries to maintain time restrictions even though intermediate references have been lost.

The method proposed here to perform the tracking does not employ temporal references. The time-independent behaviour of the system is crucial to develop interactions between the robot and its workspace. In this paper, this time-independent behaviour

This work was supported in part by the Spanish MEC under Project DPI200506222 and Grant AP2005-1458 and by the Valencian Community under Grant BPI06/015. makes the robot continue on the same path from the same point that it was tracking before the detection of a human. The method described in this paper guarantees the correct tracking in the $3 \mathrm{D}$ space. To do this, 3D information obtained from the on-line calibration of the camera is used. To sum up, the proposed system can specify a constant desired velocity during the tracking.

The location of human operators must be determined precisely in order to make possible this safe path tracking. However, most localization systems only obtain the global position of a person [12][13]. These systems do not provide information about the location of each limb of the operator's body and thus they are not suitable for close human-robot interaction. In this paper an inertial motion capture system is used because it is able to register full-body movements which can be interpreted in order to perform collaborative tasks between the robot and the human. This system is robust against occlusions [14] and magnetic interferences [15] that other motion capture technologies suffer.

This paper is organized as follows: Section II describes the proposed visual servoing scheme for tracking trajectories; in Section III the human tracking system and the algorithm employed to detect the human and move the robot away from her/him are presented; in Section IV experimental results confirm the validity of the tracking algorithm in the presence of a human and the final Section presents the main conclusions.

\section{VisUal SERVOING FOR Tracking TRAJECTORIES}

\section{A. Visual Servoing and Virtual Visual Servoing}

A visual servoing task can be described by an image function, $\mathbf{e}_{t}$, which must be regulated to 0 :

$$
\mathbf{e}_{\mathrm{t}}=\mathbf{s}-\mathbf{s}^{*}
$$

where $\mathbf{s}$ is a $\mathrm{M} \times 1$ vector containing $M$ visual features corresponding to the current state, while $\mathbf{s}^{*}$ denotes the visual features values in the desired state.

$\mathbf{L}_{\mathrm{s}}$ represents the interaction matrix which relates the variations in the image with the variations in the velocity of the camera:

$$
\dot{\mathbf{s}}=\mathbf{L}_{\mathrm{s}} \cdot \dot{\mathbf{r}}
$$

where $\dot{\mathbf{r}}$ indicates the velocity of the camera. 
By imposing an exponential decrease of $\mathbf{e}_{\mathrm{t}}\left(\dot{\mathbf{e}}_{\mathrm{t}}=-\lambda_{\mathrm{l}} \mathbf{e}_{\mathrm{t}}\right)$ it is possible to obtain the following control action for a classical image-based visual servoing [16]:

$$
\mathbf{v}_{\mathrm{c}}=-\lambda_{1} \hat{\mathbf{L}}_{\mathrm{s}}^{+}\left(\mathbf{s}-\mathbf{s}^{*}\right)
$$

where $\hat{\mathbf{L}}_{\mathrm{S}}^{+}$is the pseudoinverse of an approximation of the interaction matrix [17].

The virtual visual servoing approach [18] is based on the previous mentioned image-based visual servoing scheme. In virtual visual servoing, a virtual camera is positioned in the scene. From an image acquired by a real camera (the reference image), virtual visual servoing works moving the virtual camera iteratively. The goal is to obtain the location of the virtual camera from which the object is observed at the same position as in the reference image. In order to define the virtual visual servoing system, we assume that the observed features in the reference image are denoted by $\mathbf{p}_{\mathrm{d}}$ and $\mathbf{p}$ are the current positions of the image features projected using the camera intrinsic parameters, $\xi$, and the current extrinsic parameters, ${ }^{\mathrm{c}} \mathbf{M}_{\mathrm{o}}$ (pose of the object frame with respect to the camera frame).

In order to determine the camera extrinsic parameters, it is necessary to minimize iteratively the error, e, between the observed data, $\mathbf{p}_{\mathrm{d}}$, and the position of the same features $\mathbf{p}$ computed using the following equation:

$$
\mathbf{p}=p r_{\xi}\left({ }^{\mathrm{c}} \mathbf{M}_{\mathrm{o}}{ }^{\mathrm{o}} \mathbf{P}\right)
$$

where ${ }^{\circ} \mathbf{P}$ are the $3 \mathrm{D}$ coordinates (with respect to the object coordinate frame) of the points extracted by the camera and $p r_{\xi}$ denotes the perspective projection model according to the intrinsic parameters. From the previous defined error, e, it is possible to obtain (supposing that the camera intrinsic parameters do not vary):

$$
\dot{\mathbf{e}}=\dot{\mathbf{p}}-\dot{\mathbf{p}}_{\mathrm{d}}=\frac{\partial \mathbf{p}}{\partial \mathbf{r}} \frac{\mathrm{d} \mathbf{r}}{\mathrm{d} \mathbf{t}}
$$

where $\mathbf{r}$ is the camera pose. Equation (5) can be rewritten as:

$$
\dot{\mathbf{e}}=\mathbf{L}_{\mathrm{p}} \mathbf{v}
$$

where $\mathbf{v}$ is the instantaneous virtual camera velocity and $\mathbf{L}_{\mathrm{p}}$ the interaction matrix related to $\mathbf{p}$ [18].

To make e decrease exponentially to $0\left(\dot{\mathbf{e}}=-\lambda_{2} \mathbf{e}\right)$ the following control law is obtained:

$$
\mathbf{v}=-\lambda_{2} \mathbf{L}_{\mathrm{p}}^{+} \mathbf{e}
$$

Once $\mathbf{e}=0$ the extrinsic camera parameters are obtained. This basic scheme of the virtual visual servoing approach is enhanced in [19], where virtual visual servoing is used to compute not only the extrinsic parameters of the real camera, but also the intrinsic ones during a visual servoing task. In this way, virtual visual servoing can obtain the calibration of the camera and its relative position with reference to an object in the image. In a visual servoing task, the use of virtual visual servoing before each iteration of the control loop improves the system behaviour.

\section{B. Image trajectory to be tracked}

From an off-line stage, the discrete trajectory of the features in the image to be tracked by the robot is obtained $\boldsymbol{T}=\left\{{ }^{\mathrm{k}} \mathbf{s} / \mathrm{k} \in 1 . . \mathrm{N}\right\}$, with ${ }^{\mathrm{k}} \mathbf{s}$ being the set of $\mathrm{M}$ points or features observed by the camera at instant $\mathrm{k},{ }^{\mathrm{k}} \mathrm{s}=\left\{{ }^{\mathrm{k}} \boldsymbol{f}_{\mathrm{i}} / \mathrm{i} \in 1\right.$...M $\}$. Fig. 1 shows the image trajectory in order to illustrate the previous mentioned notation.

\section{Time-dependent methods for tracking image paths using visual servoing}

The systems proposed up to now to track trajectories in the image using visual servoing [8][9][10][11] have a temporal reference:

$$
\mathbf{v}_{\mathrm{c}}=-\lambda_{1} \hat{\mathbf{L}}_{\mathrm{s}}^{+}\left(\mathbf{s}-\mathbf{s}^{*}(\mathrm{t})\right)
$$

These systems do not guarantee the correct tracking of the path when the robot interacts with its environment. The references sent by the system can be too restrictive. In this way, the system will try to maintain these temporal restrictions even though it does not perform a correct tracking.

Stopping the time when the path tracking is interrupted could resolve the problem. But this fact implies changing the control law, as the system is not able to assure the moment when the robot has returned to the path to continue sending references.

\section{Time-independent method for tracking image paths using visual servoing}

The method developed to track trajectories in the image must resolve the problems found in the methods proposed up to now. Firstly, it must present a time-independent behaviour. Only two

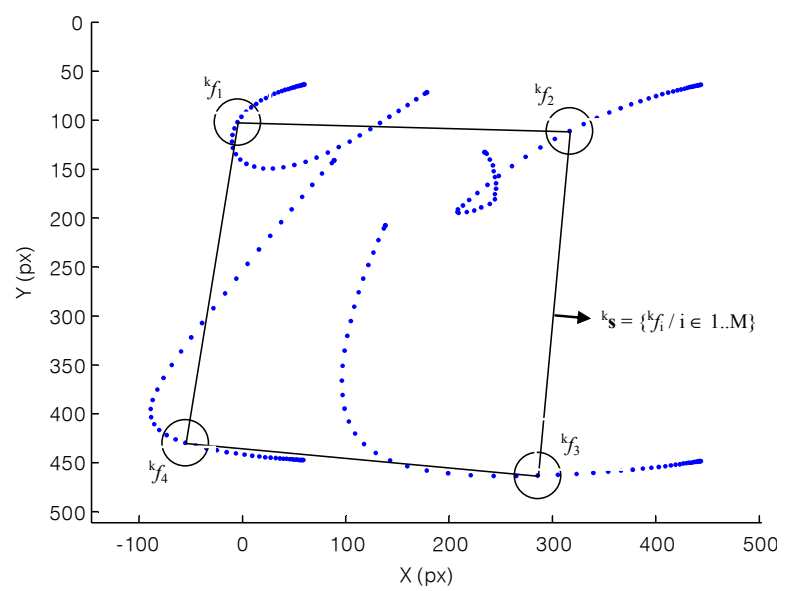

Fig. 1. Image trajectory to be tracked, $\boldsymbol{T}=\left\{{ }^{\mathrm{k}} \mathbf{s} / \mathrm{k} \in 1 . . \mathrm{N}\right\}$. 
techniques have been found in the literature with this timeindependent behaviour when tracking an image path using visual servoing [20][21]. In [20], the visual servoing system proposed to track the trajectory in the image moves the robot to a position in the path by using a classical image-based visual servoing control law. Once the error is annulled (the robot velocity is 0 ), the system guides the robot to the next position in the path. This process is repeated until the robot has visited all the references in the path. Thus, this system is not able to maintain a constant velocity during the tracking. In [21] movement flow-based visual servoing is presented. This approach employs the concept of movement flow. The movement flow is a vector field that indicates the direction in which the desired features to be used by an image-based visual servoing system must be located. In this way, it allows the tracking of the trajectory. This approach has several problems which must be solved in order to be applied in a real workspace. The movement flow-based visual servoing system has a correct behaviour in the $3 \mathrm{D}$ space. However, when the velocity is increased this behaviour is not guaranteed. Moreover, it is not possible to specify the desired velocity for doing the tracking. Another problem is that the system has an oscillatory behaviour when difficult trajectories are being tracked.

To solve the problems detected in these previous timeindependent methods, the proposed method must be able to control the desired tracking velocity.

The set of visual features observed at the initial camera position are represented by ${ }^{1} \mathbf{s}$. From this initial set of image features it is necessary to find an image configuration which provides the robot with the desired velocity, $\left|\mathbf{v}_{\mathrm{d}}\right|$. To do this, the system iterates over the set $\boldsymbol{T}$. For each image configuration ${ }^{\mathrm{k}} \mathbf{s}$ the corresponding camera velocity is determined considering an image-based visual servoing system (at this first stage $\mathbf{s}={ }^{1} \mathbf{s}$ ):

$$
{ }^{\mathrm{k}} \mathbf{v}=-\lambda_{1} \hat{\mathbf{L}}_{\mathrm{s}}^{+}\left(\mathbf{s}-{ }^{\mathrm{k}} \mathbf{s}\right)
$$

This process continues until ||$^{\mathrm{k}} \mathbf{v} \mid$ is greater than the desired velocity, $\left|\mathbf{v}_{\mathrm{d}}\right|$. At this moment, the set of features ${ }^{\mathrm{k}} \mathbf{s}$ will be the desired features to be used by an image-based visual servoing system (see (3)). However, the visual features which provide the desired velocity are between ${ }^{\mathrm{k}} \mathbf{s}$ and ${ }^{\mathrm{k}-1} \mathbf{s}$. To obtain the correct image features, the method described in the next section is proposed.

Therefore, once the control law represented in (3) is executed, the system searches again for a new image configuration which provides the desired velocity. This process continues until the complete trajectory is tracked.

\section{E. Trajectory interpolation based on virtual visual servoing to obtain the desired image features}

In order to obtain the desired velocity during the tracking, $\left|\mathbf{v}_{\mathrm{d}}\right|$, it is necessary to find a $3 \mathrm{D}$ camera location, ${ }^{\mathrm{c}} \mathbf{M}_{\mathrm{oj}}$, from which a set of features ${ }^{\mathrm{j}} \mathbf{s}$ are extracted, so that applying the control law (3) the velocity $\left|\mathbf{v}_{\mathrm{d}}\right|$ is obtained. As it is described in the previous section, these visual features will be extracted between the set of features ${ }^{\mathrm{k}} \mathbf{s}$ and ${ }^{\mathrm{k}-1} \mathbf{s}$. The problem addressed in this section is to determine the $3 \mathrm{D}$ location ${ }^{\mathrm{c}} \mathbf{M}_{\mathrm{oj}}$.

The camera extrinsic parameters ${ }^{\mathrm{c}} \mathbf{M}_{\mathrm{ok}}$ and ${ }^{\mathrm{c}} \mathbf{M}_{\mathrm{ok}-1}$ are obtained from the set of features ${ }^{\mathrm{k}} \mathbf{s}$ and ${ }^{\mathrm{k}-1} \mathbf{s}$ respectively by using virtual visual servoing (see Section II.A). From ${ }^{\mathrm{c}} \mathbf{M}_{\mathrm{ok}}$ and ${ }^{\mathrm{c}} \mathbf{M}_{\mathrm{ok-1}}$ the translations ${ }^{c} \mathbf{t}_{\mathrm{ok}}$ and ${ }^{\mathrm{c}} \mathbf{t}_{\mathrm{ok}-1}$ can be easily extracted. A linear interpolation between both translations can be obtained using the following equation:

$$
{ }^{\mathrm{c}} \mathbf{t}_{\mathrm{o} \alpha}=\alpha \cdot\left({ }^{\mathrm{c}} \mathbf{t}_{\mathrm{ok}}-{ }^{\mathrm{c}} \mathbf{t}_{\mathrm{ok}-1}\right)
$$

with $0 \leq \alpha \leq 1$. Furthermore, from ${ }^{\mathrm{c}} \mathbf{M}_{\mathrm{ok}}$ and ${ }^{\mathrm{c}} \mathbf{M}_{\mathrm{ok}-1}$ the rotations ${ }^{\mathrm{c}} \mathbf{R}_{\mathrm{ok}}$ and ${ }^{\mathrm{c}} \mathbf{R}_{\mathrm{ok}-1}$ can also be extracted. In order to develop the linear interpolation for the orientation it is first necessary to represent the previous rotations by the quaternions ${ }^{\mathrm{c}} \mathbf{Q}_{\mathrm{ok}}$ and

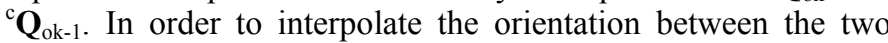
quaternions, spherical linear interpolation is used as described in [22], obtaining ${ }^{\mathrm{c}} \mathbf{Q}_{\mathrm{o \alpha}}$ :

$$
{ }^{\mathrm{c}} \mathbf{Q}_{\mathrm{o} \alpha}=\frac{{ }^{\mathrm{c}} \mathbf{Q}_{\mathrm{ok}-1} \cdot \sin ((1-\alpha) \theta)+{ }^{\mathrm{c}} \mathbf{Q}_{\mathrm{ok}} \cdot \sin (\alpha \theta)}{\sin \theta}
$$

where $\theta$ is the angle between the orientation of the camera in $\mathrm{k}-1$ and the orientation in $\mathrm{k}$. After the computation of ${ }^{\mathrm{C}} \mathbf{Q}_{\mathrm{o \alpha}}$, the rotation matrix ${ }^{\mathrm{c}} \mathbf{R}_{\mathrm{o} \alpha}$ is obtained by transforming now the quaternion.

Once the linear interpolation ${ }^{\mathrm{c}} \mathbf{M}_{\mathrm{o}}(\alpha)=\left[{ }^{\mathrm{c}} \mathbf{R}_{\mathrm{o} \alpha}{ }^{\mathrm{c}} \mathbf{t}_{\mathrm{o} \alpha}\right]$ is generated, the location ${ }^{\mathrm{c}} \mathbf{M}_{\mathrm{oj}}$ is obtained by non linear least squares optimization. To do so, it is necessary to minimize the error $\mathbf{e}_{\mathrm{o}}=\left|\mu_{\mathrm{c}}(\alpha)\right|-\left|\mathrm{v}_{\mathrm{d}}\right|$ where:

$$
\mu_{\mathrm{c}}(\alpha)=-\lambda_{1} \hat{\mathbf{L}}_{\mathrm{s}}^{+}\left(\mathbf{s}-\mathbf{s}^{*}(\alpha)\right)
$$

and

$$
\mathbf{s}^{*}(\alpha)=p r_{\xi}\left({ }^{\mathrm{c}} \mathbf{M}_{\mathrm{o}}(\alpha)^{\mathrm{o}} \mathbf{P}\right)
$$

where ${ }^{\circ} \mathbf{P}$ are the set of object points considered and $p r_{\xi}$ denotes the perspective projection model according to the intrinsic parameters, $\xi$.

Once the value $\alpha$ which minimizes $\mathbf{e}_{\mathrm{o}}$ is obtained, the set of features ${ }^{\mathrm{j}} \mathbf{s}$ will be equal to $\mathbf{s}^{*}(\alpha)$.

\section{HUMAN-ROBOT INTERACTION}

\section{A. Human Tracking}

An inertial motion capture system is used to localize precisely the human operator who may interact with the robot in the workspace. Motion capture systems are able to determine each limb location by measuring relative distances and angles between markers (or sensors) which are worn on the body. The inertial motion capture system used in this work is composed of a lycra suit with 18 IMUs (Inertial Measurement Units) on it. 
Each IMU [23] contains three gyroscopes, three accelerometers and three magnetometers which are used to obtain the orientation (Euler angles) of the limb to which the IMU is attached. The difference between the orientations of two consecutive limbs will determine the rotation of the joint which is between them. Joints' rotations calculated this way are applied over 25 nodes of a 3D hierarchical skeleton which represents the structure of the human body. The absolute position and orientation of the person in the environment is represented by the hips node which is the root node of the skeleton structure. Measurements of the other nodes will be relative rotations from this root node.

This inertial motion capture system has several advantages over other motion capture technologies [24]. The system is selfcontained and a special set-up in the workspace is not needed. Furthermore, it suffers neither from occlusions like optical systems [14] nor from important magnetic interferences like magnetic trackers [15]. Rotational measurements are very precise and they are obtained with high frequencies $(30-120 \mathrm{~Hz})$. Nevertheless, the absolute position of the skeleton (hips node position) accumulates an important error through time which is too high to locate a human operator who works near industrial robots. An additional location system is needed in order to correct this drift.

An Ultra-WideBand (UWB) radio localization system is included in the human tracking system. This UWB system is able to position more precisely operators in the workspace because it does not accumulate any drift. The human operator carries a small tag which sends UWB pulses to four sensors which are installed at fixed positions in the workspace. The angle and time of arrival of these UWB signals are used to calculate the absolute position of the operator.

The global translational measurement from the UWB system is fused with the global translation from the motion capture suit by a Kalman filter (see [25] for more details about the fusion algorithm). The resulting global position from the fusion algorithm is applied to the root node of the skeleton. Thereby, the advantages of both systems are combined: the high translational accuracy of the UWB measurements and the high frequency and accuracy of the rotational measurements from the motion capture system. The human operator will wear both systems while she/he is collaborating with the robot. Thereby, the operator is completely localized and thus the nearest limb to the robot is identified in order to develop human-robot interaction tasks.

\section{B. Safety behaviour for human-robot interaction}

All measurements from the human tracking system are registered in the computer where the robot controller is running. Every time the human tracking measurements are received, it calculates the Euclidean distance from every node of the skeleton to the end-effector of the robot. While this distance is greater than a safety threshold, the robot will continue tracking the planned path by visual-servoing. When the distance between one node and the robot is smaller than the safety threshold, the robot will stop tracking the initial path and will move away from the human operator in a linear path. When the distance of all skeleton nodes returns to be greater than the threshold, the visual servoing tracking will be re-activated to follow the original path.

\section{RESULTS}

\section{A. Experimental setup}

The system architecture (Fig. 2) is composed of an eye-inhand PHOTONFOCUS MV-D752-160-CL-8 camera at the endeffector of a 7 d.o.f. Mitsubishi PA-10 robot. The camera is able to acquire and to process up to 100 frames/second using an image resolution of $320 \times 240$. In this paper we are not interested in image processing issues; therefore, the image trajectory is generated using four laser points projected on the floor which will be the extracted features (i.e. $M=4$ ).

The human tracking system is composed by an inertial motion capture system GypsyGyro-18 from Animazoo and a UWB localization system from Ubisense. The human operator will wear both systems (motion capture suit and UWB tag) while she/he is working in the robot workplace.

\section{B. Time-independent image path tracking}

The robot employs the strategy described in Section III.B to stop the trajectory tracking when a human is too close. In these conditions the system described in Section II is employed to carry out the tracking of the desired image trajectory. In this experiment, if there is no human detection and the timeindependent system to track trajectories in the image acts normally, the tracking is 12 seconds long. However, in order to expose the difference between the system behaviour and a timedependent system, Fig. 3 shows an example of a tracking in which within the trajectory the robot detects the human stopping the tracking for approximately 3 seconds. During the presence of the human, the robot goes off the $3 \mathrm{D}$ desired path to maintain the safety distance $(1 \mathrm{~m})$. In Fig. 3 it can be observed that, once the human detection ends, the system continues with the path tracking from the next reference point in the desired path.

Let us suppose now that a visual servoing system with temporal references is used to perform the tracking of the same image trajectory. As in the previous mentioned case, within the tracking of the path, the human approaches the robot. The obtained tracking is shown in Fig. 4. The tracking system with time-dependent behaviour is not able to continue in the desired trajectory once the risk of collision with the human disappears. This is due to the fact that the temporal references are out of

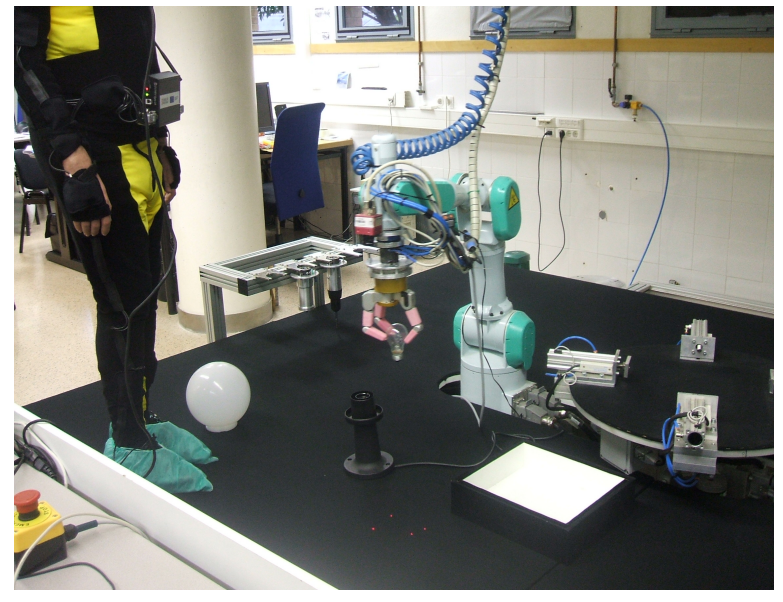

Fig. 2. Experimental setup: human tracking system (left), PA-10 manipulator (center) and visual servoing features (bottom center). 


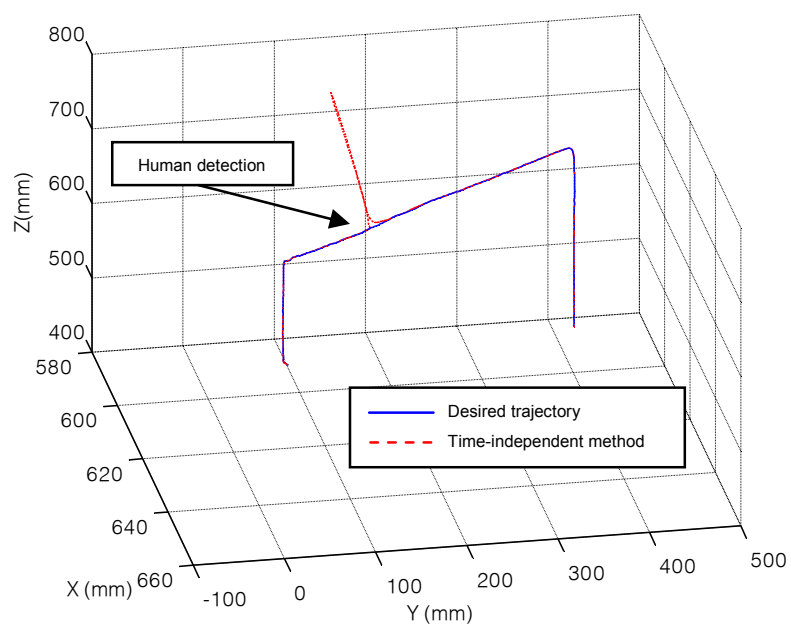

Fig. 3. 3D trajectory of the end-effector of the robot obtained with the proposed time-independent method.

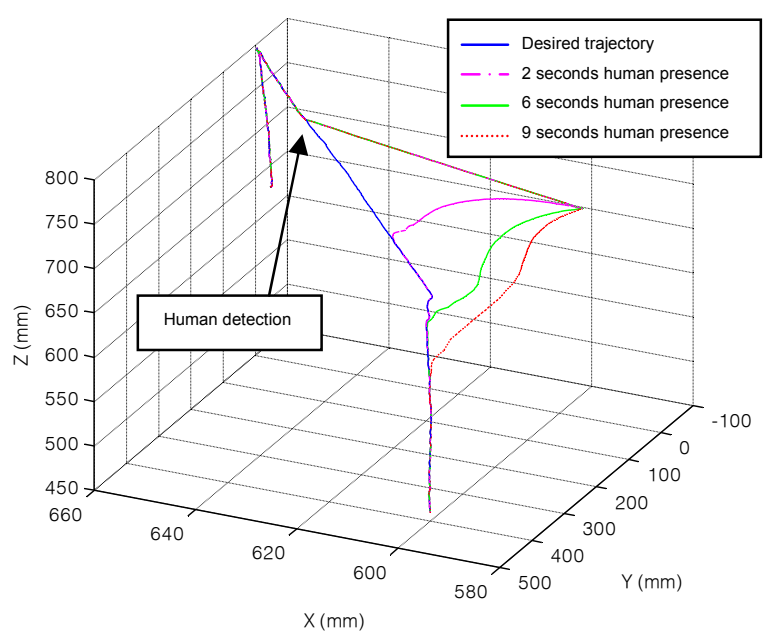

Fig. 4. 3D trajectory of the end-effector of the robot obtained with a timedependent method depending on the period of time while the human-robot distance is smaller than the safety threshold.

phase and become excessively restrictive. The system tries to maintain these temporal restrictions but it cannot perform the correct tracking of the desired trajectory.

In Fig. 4 we can observe different trajectories depending on the time that the human remains in the proximities of the robot. The figure shows clearly how the tracking makes worst as the time of the obstruction increases.

Fig. 5 shows the evolution of one of the features in the image obtained with the time-independent method described and with a time-dependent method. The tracking is correctly performed until the moment when the obstruction begins. Nevertheless, from the moment when the robot is released, the time-dependent system is not able to return to the exact point in the trajectory in which the obstruction began. This is due to the loss of the temporal references. Therefore, the time-independent method described is adequate for the tracking of image paths in tasks in which the robot interacts with a human or with any object of its workspace.
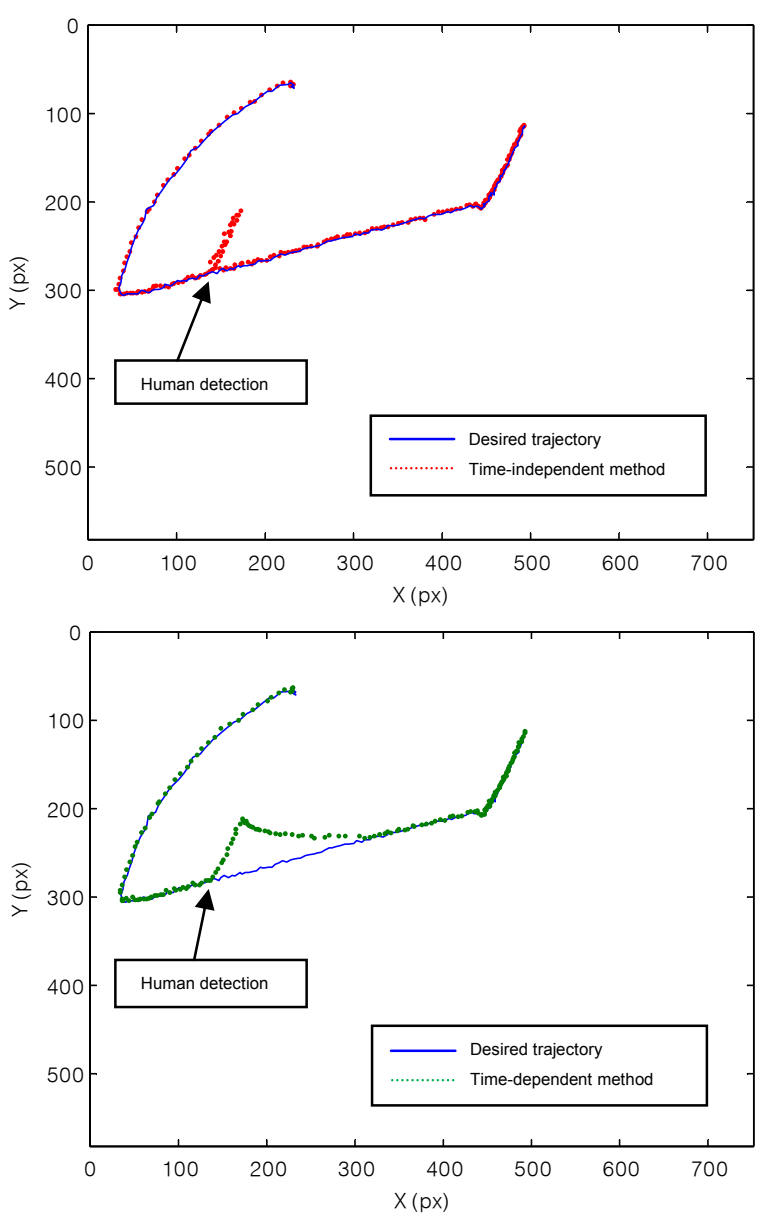

Fig. 5. Evolution of one of the features in the image space using the timeindependent tracking (top) and using a time-dependent method (bottom)

\section{Human detection during the path tracking}

The presence of the human inside the robot zone takes the robot away from the desired path. Fig. 6 shows the evolution of the distance between the nearest node of the skeleton of the human and the end-effector of the robot. It also shows the evolution of the error in 3D between the location of the endeffector during the task and the desired 3D path of the endefector. As we can see in Fig. 6, when the distance is smaller than the security threshold $(1.0 \mathrm{~m})$, the $3 \mathrm{D}$ error increases as the robot is taken away from the desired path. The time-independent system reduces this error to zero once the distance exceeds the security threshold.

\section{CONCLUSIONS}

This paper develops a visual servoing path tracking system which is able to react in a safe way when a human operator is near the robotic manipulator. The implemented visual servoing method tracks a pre-established path while there is not any risk of collision. When the distance between the robot and the human is smaller than a safety threshold, the robot controller stops the path tracking and activates a safety behaviour which moves the robot away from the human and maintains the safety distance. When the collision hazard vanishes, the robot will continue following the initial path. 

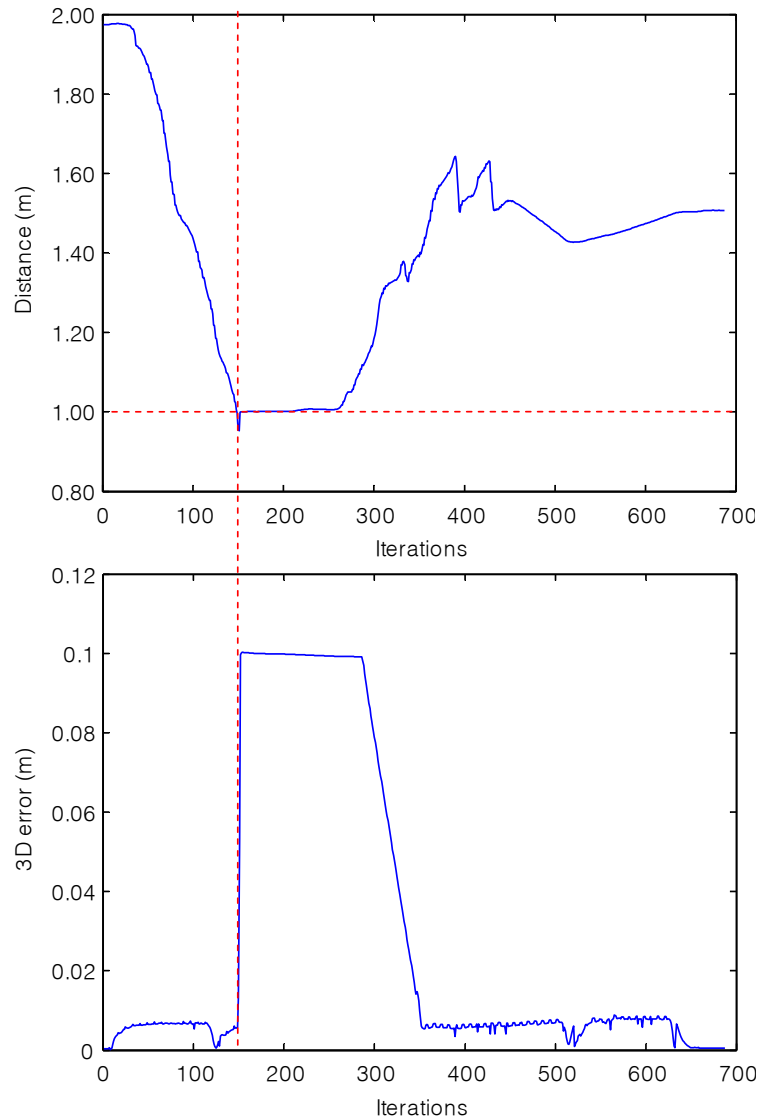

Fig. 6. Evolution of the distance between the human and the end- effector of the robot (top) and the error measured between the desired and the real trajectories of the end-effector (bottom).

The developed time-independent tracking method completes the full trajectory while previous time-dependent tracking methods lose intermediate time references and do not follow the complete trajectory.

In future work, a more detailed model of the human and of the robot structure should be studied in order to verify collisions between all the limbs of the human operator and the links of the manipulator. Thereby, more complex and precise collaboration tasks between the human and the robot will be developed.

\section{REFERENCES}

[1] Y. Yamada, Y. Hirasawa, S. Huang, Y. Umetani and K. Suita, "Humanrobot contact in the safeguarding space," IEEE/ASME Transactions on Mechatronics, vol. 2, no. 4, pp. 230-236, Dec. 1997.

[2] H. Lim and K. Tanie, "Collision-tolerant control of human-friendly robot with viscoelastic trunk," IEEE/ASME Transactions on Mechatronics, vol. 4 no. 4, pp. 417-427, Dec. 1999.

[3] S. Lu and J. H. Chung, "Collision detection enabled weighted path planning: a wrist and base force/torque sensors approach," in Proc. 12th International Conference on Advanced Robotics, Seattle, Jul. 2005, pp. 165-170.

[4] J. Heinzmann and A. Zelinsky, "Quantitative safety guarantees for physical human-robot interaction," The International Journal of Robotics Research, vol. 22, no. 7-8, pp. 479-504, Jul. 2003.

[5] O. Brock and O. Khatib, "Elastic strips: a framework for motion generation in human environments," The International Journal of Robotics Research, vol. 21, no. 12, pp. 1031-1052, Dec. 2002.
[6] P. Ogren, N. Egerstedt and X. Hu, "Reactive mobile manipulation using dynamic trajectory tracking," Proc. 2000 IEEE International Conference on Robotics and Automation, San Francisco, Apr. 2000, vol. 4, pp. 3473-3478.

[7] D. Aarno, F. Lingelbach and D. Kragid, "Constrained path planning and task-consistent path adaptation for mobile manipulators," Proc. 12th International Conference on Advanced Robotics, Seattle, Jul. 2005, pp. 268-273.

[8] G. Chesi, D. Prattichizzo, and A. Vicino, "Straight line path-planning in visual servoing," Journal of Dynamic Systems Measurement and ControlTransactions of the Asme, vol. 129, pp. 541-543, Jul 2007.

[9] G. Chesi and Y. S. Hung, "Global path-planning for constrained and optimal visual servoing," IEEE Transactions on Robotics, vol. 23, pp. 1050 1060 , Oct 2007.

[10] E. Malis, "Visual servoing invariant to changes in camera-intrinsic parameters," Ieee Transactions on Robotics and Automation, vol. 20, pp. 72-81, Feb 2004.

[11] Y. Mezouar and F. Chaumette, "Path planning for robust image-based control," IEEE Transactions on Robotics and Automation, vol. 18, no. 4 pp. 534-549, Aug. 2002.

[12] J. Hightower and G. Borriello, "Location systems for ubiquitous computing", IEEE Computer, vol. 34, no. 8, pp. 57-66, Aug. 2001.

[13] H. Liu, H. Darabi, P. Banerjee, and J. Liu, "Survey of wireless indoor positioning techniques and systems", IEEE Transactions on Systems, Man and Cybernetics Part C: Applications and Reviews, vol. 37, no. 6, pp. 1067-1080, Nov. 2007.

[14] M. Onishi, T. Odashima, Z. Luo and S. Hosoe, "An immersion-type 3D dynamic simulation environment for developing human interactive robot systems," Systems and Computers, vol. 37, no. 10, pp. 368-377, Sept. 2006.

[15] Y.H. Seo, I.W. Jeong and H.S. Yang, "Motion capture-based wearable interaction system and its application to humanoid robot, AMIO," Advanced Robotics, vol. 21, no. 15, pp. 1725-1741, Nov. 2007.

[16] F. Chaumette and S. Hutchinson. "Visual Servo Control, Part I: Basic Approaches", IEEE Robotics and Automation Magazine, vol. 13, no. 4, pp. 82-90, Dec. 2006.

[17] S. Hutchinson, G. D. Hager, and P. I. Corke, "A tutorial on visual servo control," IEEE Trans. Robotics and Automation, vol. 12, no. 5, pp. 651-670, Oct. 1996.

[18] E. Marchand and F. Chaumette, "A new formulation for non-linear camera calibration using VVS," IRISA, Rennes, France, Technical Report 1366 Jan. 2001.

[19] J. Pomares, F. Chaumette, and F. Torres, "Adaptive visual servoing by simultaneous camera calibration," in Proc 2007 IEEE International Conference on Robotics and Automation, Roma, Apr. 2007, pp. 2811-2816.

[20] F. Schramm and G. Morel. "Ensuring visibility in calibration-free path planning for image-based visual servoing," IEEE Transactions on Robotics, vol. 22, no. 4 , pp. 848-854, Aug. 2006.

[21] J. Pomares and F. Torres, "Movement-flow based visual servoing and force control fusion for manipulation tasks in unstructured environments," IEEE Transactions on Systems, Man, and Cybernetics-Part C, vol. 35, no. 1, pp. $4-15$, Jan. 2005

[22] K. Shoemake, "Animating Rotation with Quaternion Curves," $A C M$ SIGGRAPH Computer Graphics vol. 19, no 3, Jul. 1985.

[23] E. Foxlin, "Inertial head-tracker sensor fusion by a complementary separatebias Kalman filter," in Proc. IEEE Virtual Reality Annual International Symposium, Santa Clara, California, 1996, pp. 185-194.

[24] G. Welch and E. Foxlin, "Motion tracking: no silver bullet but a respectable arsenal," IEEE Computer Graphics and Applications, vol. 22, no. 6, pp. 24 38, Nov. 2002

[25] J. A. Corrales, F. A. Candelas and F. Torres, "Hybrid tracking of human operators using IMU/UWB data fusion by a Kalman filter," in Proc. $3 \mathrm{rd}$ ACM/IEEE International Conference on Human-Robot Interaction, Amsterdam, Mar. 2008, pp. 193-200. 